\title{
Phase Change Related microRNA Profiles in the Plant Regeneration Process of Avocado through Shoot-tip Culture
}

\author{
Jayeni Hiti-Bandaralage*, Alice Hayward, Chris O’Brien, Umair Ahsan, Madeline Gleeson, Yuxin Xue \\ and Neena Mitter* \\ Centre for Horticultural Science, Queensland Alliance for Agriculture and Food Innovation, The University of \\ Queensland, Brisbane, QLD, Australia \\ Email: n.mitter@uq.edu.au, uqjhitib@uq.edu.au
}

\begin{abstract}
MicroRNAs are a group of non-coding, highly conserved, small RNAs of 21-22 nucleotides found in both animals and plants. They regulate plant development by cleavage of complimentary mRNAs or translational inhibition. Avocado is a high value crop, which can be benefited by tissue culture for propagation. It has been hypothesised that tissue culture process restores juvenility in physiologically mature explants. The current study examined this by profiling two microRNAs, miR156 and miR172, involved in juvenile-to-adult phase change. The study revealed that, both miR156 and miR172 are expressed at similar levels in cultured avocado shoot meristems. MicroRNAs levels were significantly increased during the shoot elongation stage. After the acclimatization, microRNAs relative expression levels were re-established to the level of explant material used. This may suggest that the maturity state of plants, as determined by miR156 and miR172 profile, remains unchanged in propagated plants rejecting the hypothesis.
\end{abstract}

Keywords: avocado, phase change, microRNA 156/172, micropropagation.

\section{Introduction}

Mass production of economically important plant species by in vitro propagation, or tissue culture, has proven to be efficient and economical. Avocado (Persea americana Mill) is a difficult to propagate horticultural crop of high economic value, with a defined industry need for efficient clonal propagation solutions. It is grown as a grafted plant, combining agronomically important traits of the rootstock with desired post-harvest and fruit quality characteristics of the fruiting scion cultivar. Clonal rootstocks, whereby the genotype of mother plant is perfectly preserved in the offspring, are desired by industry for their uniform field performance $[1,2]$ and reportedly higher yield gains compared to that of the seedling rootstocks [3]. However, clonal propagation of avocado rootstocks is currently based on a 45-year old nursery-based technique $[4,5]$ that is arduous and time consuming, reflected by the limited availability and high price of clonal rootstocks.

In vitro propagation, or micropropagation, for clonal rootstock propagation has the advantage of producing a large number of true-to-type plantlets from few explants in a space-efficient, sterile, soil-free and stress-free environment [6-8]. The history of avocado culture in vitro runs back to the early 1970's. However, more than five decades of research has not been able to establish an industry-suitable micropropagation system to produce clonal avocado plants from physiologically mature source material due to the difficulty in root induction in vitro [9-11], until the recent developments through meristem culture by Hiti-Bandaralage et al. $[2,8]$.

A number of molecular and physiological changes may occur in tissue cultured plants over time. One effect of continuous culture is reported to be rejuvenation of tissues or plantlets to a more juvenile state $[12,13]$. This is thought to be beneficial, as juvenile avocado material in general is more amenable to shoot and root regeneration in culture [14], but would be disadvantageous if the produced plants remain in juvenile state taking longer period for flowering. With advances in molecular biology, a number of genes and regulatory factors have been implicated in the phase change of plants between juvenile and mature states, and in in vitro rejuvenated material $[12,15]$. MicroRNAs (miRNAs) are one such group of factors, which are single stranded RNA molecules approximately 22 nucleotides in length, highly involved in regulation of plant development $[16,17]$. They are highly conserved within the plant kingdom 
and control similar functions across higher plant species by repressing key developmental genes $[16,18-$ 20]. In the control of plant phase-change from juvenile to reproductive maturity, miR156 is highly expressed at the juvenile phase and gradually decreases with age, while miR172 expression is lowest in the juvenile phase but increases with plant maturity $[15,17]$. Overexpression of miR156 in Arabidopsis thaliana and Zea mays (maize) extends the juvenile vegetative characters of the plant and delays flowering, while overexpression of miR172 in Arabidopsis accelerates flowering [15]. It is also known that miR156 negatively regulates SQUAMOSA PROMOTER BINDING PROTEIN-LIKE (SPL) transcription factors to delay the onset of flowering, partly by negatively regulating miR172 [15]. This change in miR156 and miR172 abundance during the transition from juvenile to adult phase has been demonstrated in avocado [21].

The current study aimed to profile the two phase-change related microRNAs; miR156 and miR172, at different time-points during the meristem-tip culture process developed for mature avocado $[2,8]$. The intention was to understand the changes in phase-related microRNA during the continuous culture process as possible markers for rejuvenation of cultured explants. This will lead to an understanding of whether continuous in vitro culture has a role in regaining juvenile characters in mature material, which may be helpful to improve root induction of highly recalcitrant woody plant species. Further, the comparison of miRNA profiles of explants used for meristem-tip culture process with the acclimatised plants produced using this system may provide hints on the maturity state of tissue cultured plants, which is an important factor for commercial adoption. This study represents the first analysis of phaserelated miRNAs in tissue cultured avocado, with the aim to broaden our understanding of the culture process of avocado, with relevance to other woody plants.

\section{Material and Methods}

\subsection{Plant Material}

The in vitro meristem-tip culture process developed by Hiti-Bandaralage et al. [2,8] , Centre for Horticultural Science, Queensland Alliance for Agriculture and Food Innovation, University of Queensland, Australia, has several distinct culture stages. The meristem tips are subjected to several multiplication cycles (F) and several shoot elongation cycles $(\mathrm{S})$ to obtain healthy shoots for root induction. The basic difference in the two culture stages is the basal nutrient medium and hormones used to induce efficient shoot proliferation at multiplication stage, and to induce elongation of proliferated buds at shoot elongation stage.

To study the expression profiles of miR156 and miR172 during in vitro meristem-tip culture process, shoot tips were sampled from three multiplication stages (F1 - 7 days in multiplication, F3 - 3 months in multiplication and F6 - 6 months in multiplication) and four shoot elongation stages (S1 - 1 month in elongation, S3 - 3 months in elongation, S6 - 6 months in elongation and S9 - 9 months in elongation) in the sequential tissue culture process. Rooted acclimatised plants produced through the meristem-tip culture process were also sampled at 3 months from acclimatisation, which were maintained in a glasshouse at $26{ }^{\circ} \mathrm{C}$ under natural light conditions at The University of Queensland, Australia.

To determine the baseline expression levels of two microRNAs of interest in mature explants used for culture induction, shoot tips were collected from mother plants maintained in a glasshouse at $26{ }^{\circ} \mathrm{C}$ under natural light conditions at The University of Queensland, Australia. Shoot tips from juvenile seedling plants (3 months old) were also sampled to compare miR156 and miR172 expression levels with mature plants.

All shoot tips (with no leaves) were harvested as pools of 3 tips from each of three plants, except for the seedlings and acclimatised samples, which comprised one tip per plant, to form 3 biological replicates per sampling point $(n=3)$. Harvested shoot tips were immediately preserved in liquid nitrogen and were stored at $-80^{\circ} \mathrm{C}$ until processing.

\subsection{Total RNA Extraction, cDNA Synthesis and qRT-PCR}

Plant tissues were ground to fine powder under liquid nitrogen and total RNA extracted using the MasterPureTM Plant RNA Purification Kit ${ }^{\circledR}$ with DNase treatment (Epicentre by Illumina, Maddison, 
WI, USA). The Plant miScript Kit (Qiagen, Limberg, Netherlands), was used to transcribe $250 \mathrm{ng} / \mu \mathrm{L}$ of RNA into miRNA-cDNA duplex according to the manufacturer's instructions. Expression of miR156 and miR172 were determined using qRT-PCR in $20 \mu \mathrm{L}$ reactions (1x QuantiTect SYBR green master mix, $0.8 \mu \mathrm{M}$ forward primer, $1 \mathrm{x}$ miScript universal reverse primer and $2 \mu \mathrm{L}$ template $(1 / 11$ dilution)) in technical duplicate (Table 1). Thermal cycling was $95{ }^{\circ} \mathrm{C}$ for $15 \mathrm{~min}$ followed by 40 cycles of $94{ }^{\circ} \mathrm{C}$ for 30 seconds, $55{ }^{\circ} \mathrm{C}$ for 30 seconds, and $70{ }^{\circ} \mathrm{C}$ for 30 seconds. The SnoR41Y gene was used as the housekeeping gene. Melt curve analysis was included for all products in all runs. PCR efficiency was determined using LinReg software (University of Amsterdam, The Netherlands) and reactions with R2 values less than 0.995 were excluded from analysis. Cycle threshold (CT) values and LinReg PCR efficiency values were averaged for technical replicates, and relative expression (RE) to snoR41Y for miR156 and miR172 were calculated for each biological replicate $(n=3)$. Descriptive statistics were generated and one-way analysis of variance (ANOVA) was performed at confidence level of 0.05 using the statistical software IBM SPSS 23. Statistical significance for multiple comparisons were carried out with LSD tests.

Table 1. Primer sequences used for qRT-PCR

\begin{tabular}{lll}
\hline Gene & Forward primer & Reverse primer \\
\hline miR156 & 5'-TGA CAG AAG AGA GTG AGC AC & 5'- miScript Universal Reverse \\
miR172 & 5'-AGA ATC TTG ATG ATG CTG CAT & 5'- miScript Universal Reverse \\
SnoR41Y & 5'-ATA CCT CCT ACT CAT TCT GAG TC & 5'- miScript Universal Reverse \\
\hline
\end{tabular}

\section{Results}

The phase-change related microRNAs; miR156 and miR172, were profiled in shoot tips (all leaves removed) of; glasshouse grown juvenile avocado seedlings (3 months old), mature plants (used as explants for tissue culture), in vitro plants at multiplication (F1, F3 and F6 stages) and shoot elongation (S1, S3, S6 and S9 stages) and acclimatised avocado plants. The relative expression (RE) of both miR156 $(\mathrm{P}=0.012)$ and miR172 $(\mathrm{P}=0.011)$ was significantly higher in juvenile shoot tips compared to mature shoot tips of avocado (Fig. 1). Both juvenile and adult shoot tip tissues had higher relative abundance of miR156 (juvenile - 1.27, mature - 0.99) than miR172 (juvenile - 0.59, mature - 0.27), taking into account their relative primer efficiencies.

When explant material was subjected to the meristem-tip culture process, the initial abundance of miR156 (at F1 and F3 multiplication stages) remained unchanged (Fig. 2). However, at F6 a significant reduction $(\mathrm{P}=0.021)$ in miR156 was seen. Interestingly, upon transfer of $\mathrm{F} 6$ shoot tips to the first elongation stage (S1), miR156 abundance significantly increased more than 30 -fold $(\mathrm{P}<0.001)$, consistent with possible increased juvenile identity at this stage. However, this increase was not maintained in subsequent S stages (S3, S6 and S9), in which miR156 level decreased to explant levels and remained relatively constant. When comparing all the in vitro phases, the first three shoot elongation stages (S1, S3 and S6) had significantly higher miR156 abundance compared to the three stages of multiplication (F1, F3 and F6). The acclimatised plants recorded significantly less $(\mathrm{P}=0.000-0.022)$ miR156 than all shoot elongation stages analysed and had a similar miR156 profile to the initial mature explants and multiplication stages (F1, F2 and F3) (Fig. 2).

Interestingly, miR172 expression levels significantly decreased more than five-fold after 7 days $(\mathrm{P}=0.024)$ of culture $(\mathrm{F} 1)$ and remained at this low level for six months in multiplication $(\mathrm{P}=0.019)$ (Fig. 3). As miR172 is normally associated with maturity and the transition of meristems to floral identity [22], this may support the hypothesis that culture induction and multiplication may promote vegetative meristem identity and juvenility in cultured avocado shoots via miR172 repression. In general, however, relative expression of miR172 shared a similar pattern to miR156. Its abundance did not significantly change during multiplication and was significantly increased $(\mathrm{P}<0.001 ;>80$-fold $)$ during the transition from F6 to shoot elongation (S1). In contrast to miR156, however, the greatest miR172 abundance was seen for acclimatised tissue cultured plants, which was significantly greater than all the sampling time-points including the mature explants. Further investigation with additional time-points 
and target gene quantification, as well as correlation to physiological indicators of juvenility, are needed to help elucidate the pathways involved in this process further.

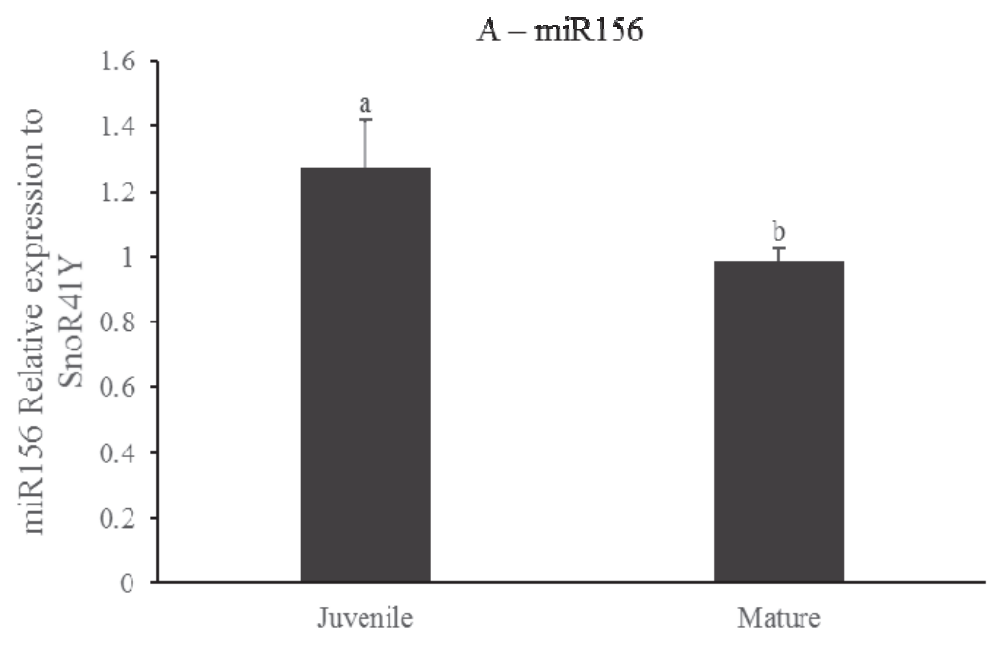

$\mathrm{B}-\operatorname{miR} 172$

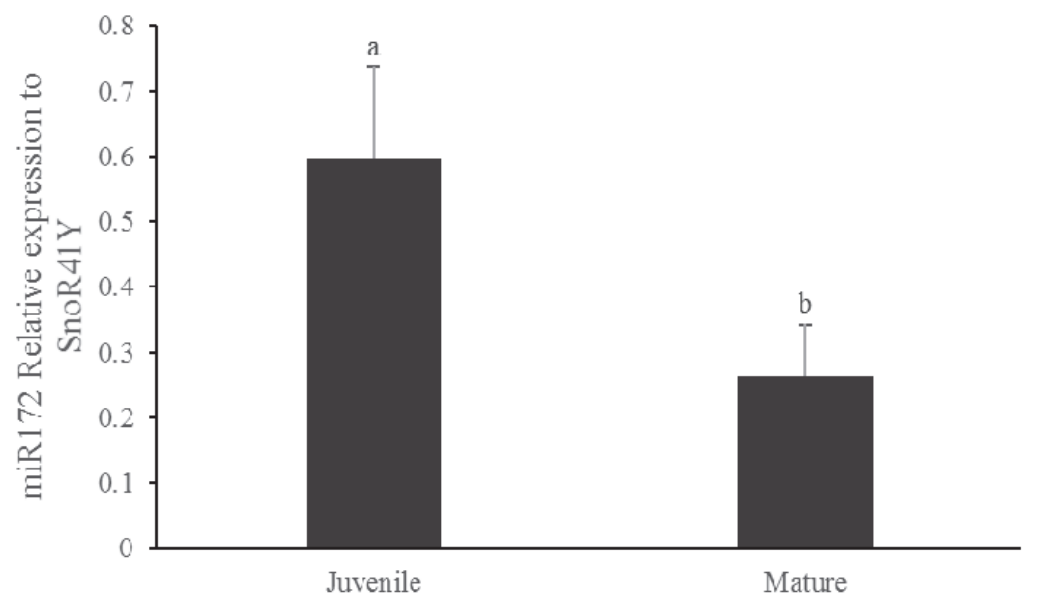

Figure 1. Both miR156 (A) and miR172 (B) relative expression were significantly less in mature shoot tips of avocado cv. 'Velvick' than in juvenile (3 months old seedlings) shoot tips. Both mature and juvenile plants were maintained in similar environmental conditions in the glasshouse prior harvesting shoot tips for RNA extraction. Expression levels are relative to housekeeping gene SnoR41Y. Error bars are means +/- SE (n=3). Significantly different values are represented by different letters $(\mathrm{P}=0.05)$. 


\section{RE miR156}

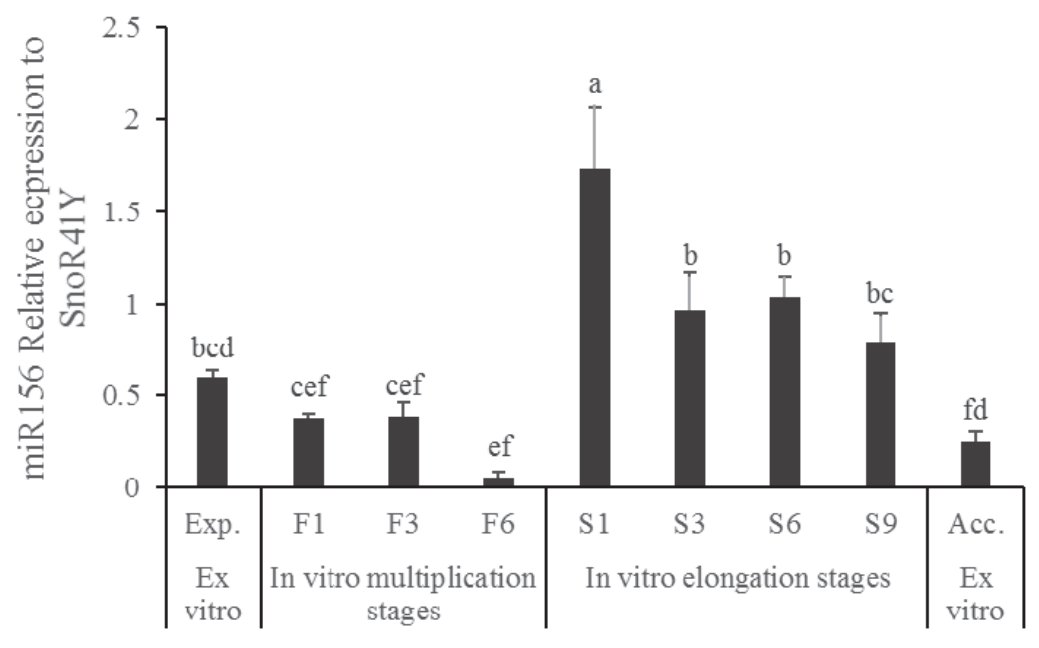

Figure 2. MiR156 relative expression levels in shoot tips of cv. 'Velvick' changed over the sampling points from explants through in vitro culture to acclimatisation stage. The initial abundance of miR156 (at F1 and F3 multiplication stages) remained unchanged to explant but significantly reduced at F6. Upon transfer of F6 shoot tips to the first elongation stage (S1), miR156 abundance significantly increased but was not maintained in subsequent S stages (S3, S6 and S9) decreasing miR156 level to explant levels. The acclimatised plants recorded significantly less miR156 than all shoot elongation stages analysed and had a similar miR156 profile to the initial mature explants and multiplication stages (F1, F2 and F3). MiR156 expression is relative to SnoR41Y housekeeping gene in mature explants before culturing (Exp.), shoots at in vitro multiplication stages (F1-7 days, F2-3 months and F6-6 months), shoots at in vitro shoot elongation stages (S1-1 months, S2-3 months, S3-6 months and S9-9 months), and acclimatised tissue culture plants (Acc.). Error bars are means $+/-\mathrm{SE}(\mathrm{n}=3)$. Values that share at least one similar letter ( $\mathrm{a}, \mathrm{b}, \mathrm{c}, \mathrm{d}, \mathrm{e}$, and $\mathrm{f}$ ) are significantly similar at $\mathrm{P}=0.05$.

\section{RE miR172}

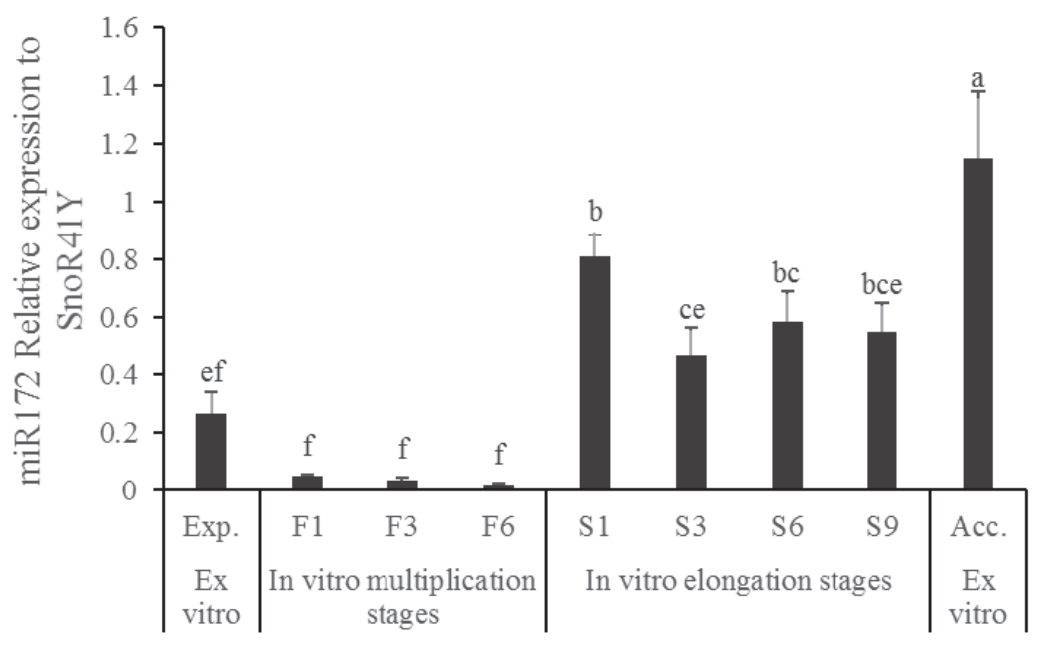

Figure 3. MiR172 relative expression levels in shoot tips of cv. 'Velvick' changed over the sampling points from explants through in vitro culture to acclimatisation stage. The initial abundance of miR172 (at F1, F3 and F6 multiplication stages) remained unchanged to explant. Upon transfer of F6 shoot tips to the first elongation stage (S1), miR172 abundance significantly increased but was not maintained in subsequent S stages (S3, S6 and S9) decreasing miR156 level to explant levels. The acclimatised plants recorded significantly high miR172 expression level than all shoot elongation and multiplication stages as well as miR172 profile of the initial mature explants. 
MiR172 expression is relative to SnoR41Y housekeeping gene in mature explants before culturing (Exp.), shoots at in vitro multiplication stages (F1-7 days, F2-3 months and F6-6 months), shoots at in vitro shoot elongation stages (S1-1 months, S2-3 months, S3-6 months and S9-9 months), and acclimatised tissue culture plants (Acc.). Error bars are means $+/-\mathrm{SE}(\mathrm{n}=3)$. Values that share at least one similar letter $(\mathrm{a}, \mathrm{b}, \mathrm{c}, \mathrm{d}$, e, and $\mathrm{f})$ are significantly similar at $\mathrm{P}=0.05$.

\section{Discussion}

The roles of miR156 and miR172 in juvenile to adult phase transition in plants was first proposed using loss-of-function and gain-of-function studies in mutants [15,23]. In maize, overexpression of the Corngrass1 gene, resulting in extra availability of miR156, induced juvenile characteristics in mature plants and also reduced the levels of miR172 [23]. On the other hand, a loss-of-function mutation in Glossy15 in maize, which encodes an APETALA2-LIKE transcription factor repressed by miR172, induces adult characters in juvenile leaves, indicating the role of miR172 in maturation. This phenomenon of miR156 and miR172 involvement in phase change has been most well studied in the model plant species Arabidopsis thaliana. In Arabidopsis, juvenile to adult phase change is a sequential process with miR156 acting to promote vegetative growth mainly by repressing the transcription factors SQUAMOSA PROMOTER BINDING PROTEIN LIKE(SPL) 9 and 10 required for the expression of miR172 and a number of other gene targets [15]. Thus, miR156 is highly expressed during the juvenile period and gradually decreases with time [15,23]. Accordingly, miR172 is less abundant in the juvenile phase but increases during the transition from juvenile to maturity [15,24]. It is predicted that miR172 targets APETALA2-LIKE transcription factors through a translational repression [22]. Both miR156 and miR172 also play key regulatory roles in the juvenile to adult phase change in many woody plants such as Quercus acutissima, Hedera helix, Acacia colei, Accacia confuse, Eucalyptus globulus, Eucalyptus grandis and Eucalyptus brachyphylla [25,26].

In accordance with other plants, in avocado, juvenile plants had an increased abundance of miR156 in shoot tips as compared to mature plants (Fig. 1A). Irrespective of the type of tissue sampled, high abundance of miR156 in juvenile plants compared to mature plants has been previously reported with Eucalyptus grandis in the stem [26] and Populus $x$ canadensis in fully expanded leaves [25]. Given the antagonistic role of miR172 in phase-change, the opposite pattern to miR156 was expected with respect to miR172. However, the results obtained revealed a significantly greater abundance of miR172 in juvenile shoot tips compared to mature shoot tips (Fig. 1B), similarly to miR156. The increase of miR172 levels in phase change have been previously shown with microRNA profiled from fully expanded leaves $[25,26]$, but not with shoot tip tissues. The different tissue types in a plant may have different expression levels of microRNA, and this was shown by Varkonyi-Gasic et al. [27] in Malus domestica (apple) profiling 21 different microRNAs in a variety of tissue types. In that study it was evident that miR172 was highly abundant in leaves compared to both shoot apex, stem, periderm, phloem and xylem. Therefore, the current result of higher abundance of miR172 in juvenile shoot tip tissues compared to mature shot tip tissues could be due to the type of tissue (shoot tips) sampled in this experiment. Also, this difference may have resulted due to an insufficient age gap between the juvenile (3 months) and mature plants (over 6 years) used herein. In a study by Wang et al. [25] a significantly higher abundance of miR172 was not seen between juvenile (1 month) to 4 year old plants but was prominent when samples were compared with juvenile (1 month) to 10 year old plants.

Looking at the relative expression levels of miR156 and miR172 it was observed that miR156 abundance was comparatively greater than miR172 in both juvenile and mature shoot tips (Fig. 1). A similar high abundance of miR156 compared to miR172 has also been reported in both leaves and roots of Cucumis sativus (cucumber) mature plants [28].

Phase related microRNA profiling during the meristem-tip culture process was desirable to test the hypothesis that in vitro culture induces juvenility over long periods of continuous culture. If the maturity status is changed in culture, it is also important to check whether the maturity level of the mother plant, from which the explants were harvested for propagation, is re-established in the final acclimatised plants produced. This is because if the maturity status of tissue culture plants produced is more juvenile than the material used for culturing, this may interfere with flowering time, resulting in problems under field conditions. Preliminary work done by Hiti-Bandaralage et al. [29] were promising 
with avocado. When mature avocado shoot tips were cultured in the multiplication medium (F), within a short period of 7 days a slight drop in miR156 RE was observed (Fig. 2). This reduction was significant at 6 months compared to initial levels of explants. This pattern of miR156 during the multiplication stage would suggest that the tissue is becoming more mature. However, this was not supported by the results seen for miR172 (see below).

The miR156 expression was significantly higher in shoot elongation stages as compared to all multiplication stages (Fig. 2). Elongation media contains increased levels of the plant hormones cytokinin and auxin to promote vegetative growth during shoot elongation. A study by Zhang et al. [18] revealed that the miR156-targeted SPL transcription factors are involved in reducing regenerative capacity of older plants by binding with B-type ARRs (ARABIDOPSIS RESPONSE REGULATORs) to reduce cytokinin response. Therefore, the exogenous application of high levels of cytokinin in the shoot elongation media may increase the level of miR156 to regain the high shoot regeneration capacity. On the other hand, many studies have proven an auxin and miR156 interaction in adventitious rooting $[20,30,31]$. In apple, exogenous application of auxin is suggested to induce a high expression level of miR156, necessary for adventitious root induction [31]. Therefore, the sudden increase in miR156 upon transferring to shoot elongation could be an effect of auxin in the culture medium.

Nonetheless, further subculture in elongation medium gradually decreased miR156 expression levels to a similar level seen for initial mature explants (Fig. 2). This is inconsistent with rejuvenation during the culture process. Moreover, acclimatised plants showed further reduced miR156, to levels that were significantly unchanged relative to initial explants. This suggests no change in juvenility as determined by miR156 abundance. Contrary to the results obtained with avocado, Li et al. [32] showed an increasing trend of miR156 expression in tissue cultured strawberry. Again, this disparity of results could be due to the type of tissue they used, which were leaves. However, their results showed a similar decrease in miR156 RE levels at the transition of in vitro to ex vitro during acclimatisation.

Similarly, to miR156, mature shoot tips, when cultured on multiplication medium, showed reduced miR172, which could potentially suggest early rejuvenation of the cultured tissues during multiplication through repression of miR172. However, again like miR156, miR172 was significantly increased from multiplication phase to shoot elongation. This is not consistent with any rejuvenation happening during the extended culture process. Indeed, the relative expression of miR172 was significantly higher in acclimatised plants as compared to that in all tissue culture stages (F1 to S9) as well as in initial explants (5.7-fold higher) (Fig. 3). Starting from explant harvest, the total process of tissue culture through to acclimatisation was nearly 21 months. Thus, it could also be hypothesised that the increase in miR172 reflects the aging of the plant over the 21 months during the culture process. This clearly shows that the tissue culture plants regenerated from mature material maintain their maturity state after the tissue culture process. Further investigations of the target genes of miR156 and miR172, and discovery of additional molecular candidates involved in juvenility, will undoubtedly help better understand the change in maturity status of tissues during different in vitro culture stages and clarify the observations of this study.

\section{Conclusions}

This study revealed that shoot tip tissues of mature avocado have a higher abundance of miR156 compared to miR172. Shoot tips multiplied in culture showed a significant reduction in both miR156 and miR172 in comparison to basal expression levels at the time of harvest from the mature mother plant. Both miR156 and miR172 showed peak levels from multiplication to shoot elongation, which may have resulted from hormones used in media. The results show no strong evidence to conclude that juvenility is induced through the meristem-tip culture process, except that tissues gain high regeneration capacity during the shoot elongation stage supported by elevated expression levels of miR156. From in vitro stages to acclimatisation, miR156 levels were reduced and miR172 levels increased, attaining the expression levels of the explants used for initiating cultures. This indicates that the tissue culture plants preserve their maturity state, as determined by miR156 and miR172 profiling, during the meristem-tip culture process. To better understand and link the pattern of phase related microRNA to juvenility and mature status during the in vitro culture process, microRNA targets and physiological studies should be explored further. 
Acknowledgements. We thank the funding bodies; the Australian Research Council Linkage Programme (grant no. LP130100870) (2013), Advance Queensland Innovation Partnership Programme (grant no. 9739018-01-155-21-021268) (2017), the Department of Agriculture and Fisheries, Australia and the University of Queensland, Australia collaborating with industry partners; Primary Growth Pty Ltd, Jasper Farms Holdings Pty Ltd, Millwood Holdings Pty Ltd, T/A Delroy Orchards and Anderson Horticulture Pty Ltd., J. C. A. Hiti-Bandaralage was supported by an Australian Postgraduate Award.

\section{References}

1. Noguera, C.F., Van Rooyenē, Z. and Köhneē, S. (2011) Reasons for the Use of Clonal Avocado Rootstocks around the World. Actas Proccedings, Cairns, Australia. http://worldavocadocongress2011.com/userfiles/file/ Consuelo\%20Fernandez\%201030-1050.pdf.

2. Hiti Bandaralage, J.C.A. (2019, June 7) Micropropagation as an Alternative for Avocado Clonal Propagation. PhD Thesis, The University of Queensland. https://doi.org/10.14264/uql.2019.531.

3. Efendi, D. and Litz, R.E. (2003) Cryopreservation of Avocado. V Congreso Mundial Del Aguacate, Actas: Consejería de Agricultura y Pesca, Junta de Andalucía. Sevilla, España, 111-114. http://www.avocadosource. com/WAC5/Papers/WAC5_p111.pdf.

4. Platt, R.G. (1976) Current Techniques of Avocado Propagation. Proceedings of the First International Tropical Fruit Short Course: The Avocado. Gainesville (FL): Florida Cooperative Extension Service and Institute of Food and Agricultural Services, University of Florida., 92-95. http://avocadosource.com/Journals/ITFSC/ PROC_1976_PG_92-95.pdf.

5. Ernst, A.A. (1999) Micro Cloning: A Multiple Cloning Technique for Avocados Using Micro Containers. Revista Chapingo Serie Horticultura, 5, 217-220.

6. Mohamed-Yasseen, Y. (1993) Morphogenesis of Avocado in Vitro. A Review. California Avocado Society Yearbook, 77, 101-105.

7. Hiti-Bandaralage, J.C.A., Hayward, A. and Mitter, N. (2017) Micropropagation of Avocado (Persea Americana Mill.). American Journal of Plant Sciences, 08, 2898. https://doi.org/10.4236/ajps.2017.811197.

8. Hiti-Bandaralage, J.C.A., Hayward, A., O'Brien, C., Gleeson, M., Nak, W. and Mitter, N. (2019) Advances in Avocado Propagation for the Sustainable Supply of Planting Materials. Achieving Sustainable Cultivation of Tropical Fruit, Burleigh Dodds Science Publishing, Cambridge, United Kingdom, 215-238. https://shop. bdspublishing.com/store/bds/detail/product/3-190-9781786765369.

9. Cooper, P.A. (1987) Advances in the Micropropagation of Avocado (Persea Americana Mill.). Acta Horticulturae, 212, 571-576.

10. Barceló-Muñoz, A., Encina, C.L., Simón-Pérez, E. and Pliego-Alfaro, F. (1999) Micropropagation of Adult Avocado. Plant cell, tissue and organ culture, 58, 11-17.

11. Bandaralage, J.C.A.H., Hayward, A., O'Brien, C. and Mitter, N. (2015) Gibberellin and Cytokinin in Synergy for a Rapid Nodal Multiplication System of Avocado. VIII World Avocado Congress, Actas Proceedings, Lima, Peru, 95-98. http://www.avocadosource.com/WAC8/Section_02/HitiBandaralageJCA2015.pdf.

12. Chen, Y.-T., Shen, C.-H., Lin, W.-D., Chu, H.-A., Huang, B.-L., Kuo, C.-I., Yeh, K.-W., Huang, L.-C. and Chang, I.-F. (2012) Small RNAs of Sequoia Sempervirens during Rejuvenation and Phase Change. Plant Biology, 15, 27-36. https://doi.org/10.1111/j.1438-8677.2012.00622.x.

13. Hiti Bandaralage, J.C.A., Hayward, A. and Mitter, N. (2019) Structural Relation for Acclimatisation Success for in Vitro Cultured Avocado. Acta Horticulturae.

14. Zirari, A. and Lionakis, S.M. (1994) Effect of Cultivar, Explant Type, Etiolation Pretreatment and the Age of Plant Material on the in Vitro Regeneration Ability of Avocado (Persea Americana). Acta Horticulturae, 365, 69-76.

15. Wu, G., Park, M.Y., Conway, S.R., Wang, J.-W., Weigel, D. and Poethig, R.S. (2009) The Sequential Action of MiR156 and MiR172 Regulates Developmental Timing in Arabidopsis. Cell, 138, 750-759. https://doi.org/ 10.1016/j.cell.2009.06.031.

16. Kidner, C.A. and Martienssen, R.A. (2005) The Developmental Role of MicroRNA in Plants. Current Opinion in Plant Biology, 8, 38-44. https://doi.org/10.1016/j.pbi.2004.11.008.

17. Huijser, P. and Schmid, M. (2011) The Control of Developmental Phase Transitions in Plants. Development, 138, 4117-4129. https://doi.org/10.1242/dev.063511. 
18. Zhang, B., Pan, X., Cannon, C.H., Cobb, G.P. and Anderson, T.A. (2006) Conservation and Divergence of Plant MicroRNA Genes. The Plant Journal, 46, 243-259. https://doi.org/10.1111/j.1365-313X.2006.02697.x.

19. Molnár, A., Schwach, F., Studholme, D.J., Thuenemann, E.C. and Baulcombe, D.C. (2007) MiRNAs Control Gene Expression in the Single-Cell Alga Chlamydomonas Reinhardtii. Nature, 447, 1126-1129. https://doi.org/ 10.1038 /nature05903.

20. Gleeson, M., Constantin, M., Carroll, B.J. and Mitter, N. (2014) MicroRNAs as Regulators of Adventitious Root Development. Journal of Plant Biochemistry and Biotechnology, 23, 339-347. https://doi.org/10.1007/ s13562-014-0269-3.

21. Ahsan, M.U., Hayward, A., Alam, M., Bandaralage, J.H., Topp, B., Beveridge, C.A. and Mitter, N. (2019) Scion Control of MiRNA Abundance and Tree Maturity in Grafted Avocado. BMC Plant Biology, 19, 382. https://doi.org/10.1186/s12870-019-1994-5.

22. Aukerman, M.J. and Sakai, H. (2003) Regulation of Flowering Time and Floral Organ Identity by a MicroRNA and Its APETALA2-Like Target Genes. The Plant Cell, 15, 2730-2741. https://doi.org/10.1105/tpc.016238.

23. Chuck, G., Cigan, A.M., Saeteurn, K. and Hake, S. (2007) The Heterochronic Maize Mutant Corngrass1 Results from Overexpression of a Tandem MicroRNA. Nature Genetics, 39, 544-549. https://doi.org/10.1038/ng2001.

24. Schwab, R., Palatnik, J.F., Riester, M., Schommer, C., Schmid, M. and Weigel, D. (2005) Specific Effects of MicroRNAs on the Plant Transcriptome. Developmental Cell, 8, 517-527. https://doi.org/10.1016/ j.devcel.2005.01.018.

25. Wang, J.-W., Park, M.Y., Wang, L.-J., Koo, Y., Chen, X.-Y., Weigel, D. and Poethig, R.S. (2011) MiRNA Control of Vegetative Phase Change in Trees. PLOS Genet, 7, e1002012. https://doi.org/10.1371/ journal.pgen.1002012.

26. Levy, A., Szwerdszarf, D., Abu-Abied, M., Mordehaev, I., Yaniv, Y., Riov, J., Arazi, T. and Sadot, E. (2014) Profiling MicroRNAs in Eucalyptus Grandis Reveals No Mutual Relationship between Alterations in MiR156 and MiR172 Expression and Adventitious Root Induction during Development. BMC Genomics, $15,524$. https://doi.org/10.1186/1471-2164-15-524.

27. Varkonyi-Gasic, E., Gould, N., Sandanayaka, M., Sutherland, P. and MacDiarmid, R.M. (2010) Characterisation of MicroRNAs from Apple (Malus Domestica 'Royal Gala') Vascular Tissue and Phloem Sap. BMC Plant Biology, 10, 159. https://doi.org/10.1186/1471-2229-10-159.

28. Mao, W., Li, Z., Xia, X., Li, Y. and Yu, J. (2012) A Combined Approach of High-Throughput Sequencing and Degradome Analysis Reveals Tissue Specific Expression of MicroRNAs and Their Targets in Cucumber. PLOS ONE, 7, e33040. https://doi.org/10.1371/journal.pone.0033040.

29. Hiti Bandaralage, J.C.A., Hayward, A., O'Brien, C. and Mitter, N. (2018) Temporal Profile of JuvenilityAssociated MicroRNAs during Tissue Culture of Avocado. Acta Horticulturae, 779-784. https://doi.org/ 10.17660/ActaHortic.2018.1205.97.

30. Yu, N., Niu, Q.-W., Ng, K.-H. and Chua, N.-H. (2015) The Role of MiR156/SPLs Modules in Arabidopsis Lateral Root Development. The Plant Journal, 83, 673-685. https://doi.org/10.1111/tpj.12919.

31. Xu, X., Li, X., Hu, X., Wu, T., Wang, Y., Xu, X., Zhang, X. and Han, Z. (2017) High MiR156 Expression Is Required for Auxin-Induced Adventitious Root Formation via MxSPL26 Independent of PINs and ARFs in Malus Xiaojinensis. Frontiers in Plant Science, 8. https://doi.org/10.3389/fpls.2017.01059.

32. Li, H., Zhao, X., Dai, H., Wu, W., Mao, W. and Zhang, Z. (2012) Tissue Culture Responsive MicroRNAs in Strawberry. Plant Molecular Biology Reporter, 30, 1047-1054. https://doi.org/10.1007/s11105-011-0406-2. 